\title{
O impacto das habilidades sociais e das estratégias de enfrentamento na resolução de problemas em universitários de psicologia
}

\section{The impact of social skills and coping strategies on problem solving in psychology university students}

\section{El impacto de las habilidades sociales y las estrategias de afrontamiento en la resolución de problemas en estudiantes universitarios de psicología}

\author{
Zeimara de Almeida Santos ${ }^{1}$, ORCID 0000-0001-8674-8738 \\ Adriana Benevides Soares ${ }^{2}$, ORCID 0000-0003-2892-1808 \\ ${ }^{12}$ Universidade Salgado de Oliveira. Brasil
}

\begin{abstract}
Resumo: Este estudo objetivou investigar se as Habilidades Sociais e Estratégias de Enfrentamento predizem a variável Resolução de Problemas em estudantes do curso de Psicologia. Participaram 409 universitários de Psicologia de instituições privadas, com idade variando de 18 a 34 anos $(M=24,97$; $D P=5,04)$. Foram utilizados como instrumentos o Inventário de Habilidades Sociais, a Escala Modos de Enfrentamento de Problemas e a Escala de Habilidades para Resolução de Problemas. Os resultados mostraram que a Escala Modos de Enfrentamento de Problemas e o Inventário de Habilidades Sociais juntos representaram 16\% da variância da escala geral da Escala de Habilidades para Resolução de Problemas, o que pode ser uma indicação de como os alunos resolveram seus problemas. Os resultados contribuem para o desenvolvimento das habilidades de solução de problemas envolvidas no treinamento de futuros psicólogos e no desenvolvimento dos estudantes.
\end{abstract}

Palavras-chave: habilidades sociais; universitários; resolução de problemas; estratégias de afrontamiento

\begin{abstract}
This study aimed to investigate whether Social Skills and Coping Strategies predict the variable Problem Solving in psychology students. Participants were 409 psychology students from private institutions, with ages ranging from 18 to 34 years $(M=24.97 ; S D=5.04)$. The Social Skills Inventory, the Problem-Solving Modes Scale and the Problem-Solving Skills Scale were used as instruments. The results showed that the Problem Coping Modes Scale and the Social Skills Inventory together accounted for $16 \%$ of the variance of the overall scale of the Problem Solving Skills Scale, which may be an indication of how students solved their problems. The results contribute to the development of problem-solving skills involved in the training of future psychologists and in the development of students.
\end{abstract}

Keywords: social skills; undergraduate students; problem solving; coping strategies 
Resumen: Este estudio tuvo como objetivo investigar si las habilidades sociales y las estrategias de afrontamiento predicen la resolución de problemas en los estudiantes de psicología. Los participantes fueron 409 estudiantes de psicología de instituciones privadas, con edades comprendidas entre 18 y 34 años $(M=24.97 ; D E=5.04)$. Se utilizaron el Inventario de habilidades sociales, la Escala de modos de resolución de problemas y la Escala de habilidades de resolución de problemas. Los resultados mostraron que la Escala de modos de resolución de problemas y el Inventario de habilidades sociales en conjunto representaron el 16\% de la varianza de la escala general de la Escala de habilidades para resolver problemas, lo que puede ser indicativo de cómo los estudiantes resolvieron sus problemas. Los resultados contribuyen al desarrollo de habilidades de resolución de problemas involucradas en la formación de futuros psicólogos y en el desarrollo de los estudiantes.

Palabras clave: habilidades sociales; universitarios; resolución de problemas; estrategias de afrontamiento

Recebido: 01/02/2018

Aceito: $13 / 06 / 2020$

\section{Como citar:}

Almeida Santos, Z., \& Soares, A.B. (2020). O impacto das habilidades sociais e das estratégias de enfrentamento na resolução de problemas em universitários de psicologia Ciencias Psicológicas, 14(2), e2228. doi: https://doi.org/10.22235/cp.v14i2.2228

Correspondência: Zeimara de Almeida Santos, Universidade Salgado de Oliveira. E-mail: zeimaradealmeida@gmail.com.Adriana Benevides Soares,e-mail: adrianabenevides@gmail.com

A formação do Psicólogo, conforme mostra o estudo de Mourão e Abbad (2016) necessita ser repensada em termos da formação de competências nas diversas regiões brasileiras onde os cursos são realizados. Além disso, a baixa qualificação dos estudantes tem sido apresentada nos relatórios do curso realizado por meio do ENADE (Exame Nacional de Desempenho dos Estudantes/ Ministério da Educação, 2018) não só no nível técnico explicitado, no desconhecimento conceitual e teórico, mas também no domínio da língua e da capacidade reflexiva. Ainda que as Diretrizes Curriculares do Curso de Psicologia apresentem características e estratégias de desenvolvimento exigidas para a profissão, Mourão e Abbad (2016) indicam déficit para enfrentar de forma adequada os processos de interação interpessoal que ainda existem na formação deste profissional.

Diante da natureza interpessoal da atuação profissional do psicólogo, fica evidente que desde a sua formação é necessário desenvolver e aprimorar competências sociais para além da aprendizagem de conteúdos acadêmicos. O manejo das relações sociais no contexto de trabalho associado à competência técnica é apontado como critério para a avaliação do papel do terapeuta (Soares, Gomes, Maia, Gomes, \& Monteiro, 2016). Esses mesmos autores consideram que a prática do psicólogo deve ser interativa para facilitar o desenvolvimento das habilidades interpessoais das pessoas e não se restringir apenas a qualificação técnica. Estudiosos (Soares \& Prette, 2015) da área de habilidades sociais complementam mostrando que a formação de terceiro grau deve articular tanto a competência técnica quanto a social, com domínio de técnicas específicas (capacidade instrumental), domínio de conhecimentos teóricos que subsidie o pensamento crítico (capacidade analítica) e o desempenho social que atenda a diferentes demandas (competência social). Portanto, a competência social é assim considerada como um requisito importante para atuação do futuro terapeuta. 
O que se pode perceber é que os cursos de graduação privilegiam basicamente o conhecimento técnico em detrimento do desenvolvimento de competências socioemocionais, cognitivas e metacognitivas (Leme et al., 2016). É no ambiente acadêmico que a formação profissional dos universitários deve estar pautada cada vez mais na competência social, além da competência técnica (Z. Del Prette, \& Del Prette, 2001).

Não obstante, o desenvolvimento da competência social tem sido uma questão pendente na formação superior, pois não são planejadas condições sistemáticas de ensino para sua promoção (Ramos, Costa, \& Feitosa, 2017). Nesse sentido, a literatura evidencia que um bom repertório de Habilidades Sociais precisa ser integrado aos domínios de competência (conhecimento, habilidades e atitudes) para o pleno funcionamento profissional. As habilidades sociais podem ser compreendidas como diferentes classes de comportamentos sociais que ajudam os indivíduos a enfrentarem os processos de interação interpessoal (A. Del Prette \& Del Prette, 2017).

Os autores Soares e Del Prette (2015) verificaram que dentre as habilidades mais deficitárias dos estudantes de Psicologia estão às habilidades assertivas de argumentar a favor de suas ideias e discordar e recusar pedidos. Soares e Z. Del Prette (2015) justificaram a importância de um bom repertório de habilidades sociais dos estudantes de Psicologia, argumentando que sua atuação se dá essencialmente por meio de relações interpessoais, sendo indispensáveis para a efetividade dessa atuação. O estudo de Tavares, Couto e Silva (2012), complementarmente mostrou que os estudantes de Psicologia apresentaram mais relações amigáveis nas relações interpessoais, porém menos manifestações assertivas de sentimentos positivos do que os demais universitários. Além disso, a pesquisa indicou que os alunos de Psicologia obtiveram escores de Sentimento Positivo $(M=21,79)$; Conversação $(M=18,80)$; Situações Novas $(M=9,78)$; Agressividade $(M=8,66)$ mais elevados de habilidades sociais do que os de outros cursos Sentimento Positivo $(M=19,25)$; Conversação $(M=17,73)$; Situações Novas $(M=7,41)$; Agressividade $(M=6,92)$ o que, segundo os autores, pode ser explicado pela formação acadêmica diferenciada e pela pressão social das expectativas de relações interpessoais de amabilidade.

Com o intuito de identificar os problemas que acarretam déficits em habilidades sociais na vida acadêmica de universitários, o estudo de Pereira, Wagner e Oliveira (2014) verificou que carências em habilidades sociais podem trazer prejuízos na prática do psicólogo, tendo em vista a relação que o profissional estabelece com seus clientes. O estudo contou com a participação de 69 estudantes de Psicologia de duas universidades privadas do Rio Grande do Sul e os resultados indicaram que os estudantes apresentaram déficits em 43,5\% das habilidades sociais, indicando baixos escores em Autoafirmação de Afeto Positivo e Autoestima, Enfrentamento e Autoafirmação com Risco.

Em uma perspectiva de fazer com que os estudantes adquiram mais habilidades sociais em situações interpessoais universitárias do relacionamento interpessoal, Lima e Soares (2015) estruturaram um treinamento de habilidades sociais a partir de situações consideradas difíceis no contexto acadêmico, visando favorecer o enfrentamento dessas situações, tendo como objetivos a aquisição de habilidades sociais pelos universitários; o fortalecimento das habilidades sociais já existentes e a extinção de comportamentos concorrentes em um caráter preventivo. Participaram deste programa onze universitários de instituições públicas e privadas de Ensino Superior do Estado do Rio de Janeiro. Além das habilidades relacionais, o contexto universitário exige autonomia na resolução de demandas acadêmicas como o cumprimento de horários, frequência nas aulas, nos estágios e a realização às avaliações, o que pode representar um momento difícil e de muitos desafios, pois terão que demonstrar responsabilidade e relacionamento adequado para que possam tomar decisões a fim de resolver seus problemas/conflitos.

Resolver problemas/conflitos é uma competência que tem sido pouco estudada nos estudantes de psicologia. As habilidades de solução de problemas são definidas por um processo metacognitivo que envolve a compreensão da natureza do problema e a identificação de soluções eficazes para a modificação da situação ou mesmo das reações à situação problemática, 
modificando as emoções negativas geradas (D'Zurilla \& Nezu, 1990). Trata-se de um processo cognitivo no qual o sujeito aprende um repertório de respostas efetivas, e desenvolve habilidades para obtenção de informações relevantes de suas ações (Monteiro \& Soares, 2016).

As reflexões sobre as habilidades de resolução de problemas em estudantes universitários motivou um estudo correlacional-descritivo (Ranjbar, Bayani, \& Bayani, 2013), que teve o propósito de verificar a relação entre a saúde mental dos estudantes da graduação (Ciencia Médica, Humanas, Agricultura, Engenharia e Arte) com a resolução de problemas sociais. Com uma amostra de 369 universitários a análise dos dados mostrou que a dificuldade de resolução de problemas sociais foi significativamente associada negativamente a sintomas somáticos, ansiedade, insônia, disfunção social e depressão grave. Nessa direção, possuir a habilidade de resolver problemas sociais possibilita uma análise de escolhas que capacita os universitários a implementarem de forma eficaz estratégias de enfrentamento para lidarem com problemas e demandas do cotidiano. A solução dos problemas aumenta a probabilidade de adaptação de enfrentamento e reduz as dificuldades cotidianas da vida (D'Zurilla, \& Nezu, 1990; 2007).

Resolver problemas envolve formas de enfrentamento e de esforço cognitivo para regular a emoção e gerir as situações, o que nos leva a definição de estratégias de enfrentamento. As estratégias de enfrentamento podem ter estilos diferentes, tais como o focado no problema, que é compreendido como um esforço em modificar a situação que originou o estresse, na emoção, que são definidas como o esforço para regular o estado emocional e no suporte social, que diz respeito ao apoio de outras pessoas, pois se espera por soluções que independem da ação do indivíduo (Santos, 2016). Em adição, as estratégias de enfrentamento têm impacto na qualidade de vida do indivíduo. Em geral, estudantes de ambos os sexos diferem na capacidade percebida para lidar com o enfrentamento de problemas. Possíveis determinantes, tais como idade, sexo, religião, faculdade e desempenho acadêmico podem influenciar o desenvolvimento da capacidade de enfrentamento de problemas, por exemplo (Gbenga \& David, 2015).

No contexto internacional, pesquisas têm investigado a forma como os graduandos enfrentam situações adversas relativas à adaptação ao Ensino Superior (Córdoba-Sánchez \& Limonero-García, 2015; Hirsch et al., 2015). As estratégias de enfrentamento baseadas no humor podem estar relacionadas a uma melhor adaptação acadêmica (Oguz-Duran \& Yuksel, 2010). Ao considerar essa estratégia adotada, esse resultado destaca a ideia de que quanto mais o aluno souber lidar com as dificuldades interpessoais, formas pessoais de lidar com eventos adversos e comportamentos inadequados, maior possibilidade de adesão a Universidade, ao curso e a escolha profissional. Similarmente, Oliveira, Carlotto, Vasconcelos e Dias, (2014) identificam possíveis relações entre adaptação acadêmica e estratégias de enfrentamento em estudantes universitários brasileiros. Os resultados indicaram que as estratégias de enfrentamento baseadas na resolução de problemas e no suporte social podem estar relacionadas com uma melhor adaptação à universidade.

Esses resultados contrastam em parte com o estudo de Reeve et al. (2013) que encontrou como desfecho que as estratégias de enfrentamento relacionadas a resolução de problemas são associadas a níveis mais baixos de estresse na universidade. Neste sentido, Carlotto, Teixeira e Dias (2015) com 412 estudantes realizaram uma pesquisa sobre adaptação acadêmica e estratégias de enfrentamento em estudantes universitários. Os autores concluíram que as estratégias focadas na emoção estiveram correlacionadas negativamente com o processo de adaptação acadêmica e a estratégia baseada no suporte social correlacionou-se de forma positiva com o processo de adaptação acadêmica, indicando que a procura de suporte pode sugerir uma peculiaridade i em lidar com os estressores de forma individual. Talvez, instrumentar o estudante para enfrentar as situações do cotidiano universitário pode trazer resultados significativos e favorecer a melhora de sua adaptação à nova realidade acadêmica.

Estendendo a discussão sobre estratégias de enfrentamento, outro estudo (Sagone \& De Caroli, 2014) também com universitários tratou das relações entre a capacidade de adaptação, as 
dimensões do bem-estar psicológico e as estratégias de enfrentamento, em uma amostra de 183 estudantes italianos com idades entre 20 a 26 anos da Universidade de Catania. Os resultados indicaram que quase todas as dimensões do bem-estar psicológico (crescimento pessoal, autonomia e relações positivas com os outros) foram correlacionadas positivamente com estratégias de enfrentamento.

Acresce ainda notar que o grande desafio para a formação do futuro psicólogo refere-se à posição dos alunos diante do conhecimento (manter-se atualizado, contextualizar os conhecimentos existentes, desenvolver a autonomia) e as características pessoais (capacidade para sensibilizar, flexibilidade interpessoal e adaptabilidade), pois constituem habilidades requeridas para o exercício profissional (Ramos et al., 2017). De maneira geral, o estudo de Soares e Z. Del Prette (2015) destaca a importância da relação interpessoal profissional de universitários de psicologia, juntamente com a identificação de dificuldades generalizadas entre os discentes. Observam-se diversas produções sobre a temática habilidades sociais e estratégia de enfrentamento, ou habilidades sociais e resolução de problemas, porém constata-se a ausência de estudos que verifique se os construtos habilidades sociais e estratégias de enfrentamento impactam no construto resolução de problemas. A literatura permite inferir que um repertório de habilidades sociais elaborado possivelmente possa contribuir para uma formação mais competente dos universitários. Convém salientar, entretanto, que a análise de predição dessas variáveis possivelmente pode facilitar na compreensão da relação de gerenciamento de situações sociais acadêmicas do aluno de psicologia em seu cotidiano. Desta forma, é justificável constatar como o os escores de estratégias de enfrentamento (conduzir as situações de estresse das questões vivenciadas nas relações interpessoais) e habilidades sociais (gerenciamento das relações interpessoais) impactam na resolução de problemas (com o fim de minimizar níveis de ansiedade e aumentar a percepção de bem-estar do aluno), além de atuar na promoção do ajustamento dos mesmos. Enfim, a partir dessa fundamentação, o presente estudo objetivou investigar se as habilidades sociais e estratégias de enfrentamento predizem a variável resolução de problemas em estudantes do curso de psicologia.

\section{Método}

\section{Participantes}

Participaram da pesquisa 409 universitários da graduação de Psicologia de instituições privadas do Estado do Rio de Janeiro, sendo a amostra não probabilística e de conveniência. Os estudantes estavam incluídos em uma faixa etária entre 18 e 34 anos $(M=24,97 ; D P=5,04)$ de ambos os sexos, sendo 323 (79\%) do sexo feminino e $86(21 \%)$ do masculino. Os participantes pertenciam à classe social A $(12 \%, n=50)$; a B1 $(20 \%, n=80) ; \mathrm{a} \mathrm{B} 2(33 \%, n=136) ; \mathrm{a} \mathrm{C} 1(26 \%$, $n=105)$; a C2 (8\%, $n=33)$; a D $(1 \%, n=5)$. De acordo com Critério de Classificação Econômica Brasil (Associação Brasileira de Empresas de Pesquisas (ABEP, 2017), considera-se o nível socioeconômico tendo em vista a posse de bens, a presença de empregada doméstica e a escolaridade do chefe da família. O escore obtido pode variar de zero a 46 pontos. Este escore foi categorizado em níveis, conforme recomendação da ABEP: A (35-46 pontos), B (23-34 pontos), $\mathrm{C}$ (14-22 pontos), D (8-13 pontos) e $\mathrm{E}$ (0-7 pontos). As classes D e $\mathrm{E}$ foram reagrupadas em uma mesma categoria, devido a sua baixa frequência.

\section{Instrumentos}

- O Inventário de Habilidades Sociais - (IHS) (Z. Del Prette, \& Del Prette, 2001) é um instrumento que se caracteriza por avaliar o desempenho social em diferentes situações. Na folha 
de resposta há uma escala do tipo Likert que varia de 0 a 4 com o significado de nunca ou raramente até sempre ou quase sempre. A aplicação do instrumento pode ser realizada de maneira coletiva ou individual. A análise das propriedades psicométricas, no estudo original obteve índices de discriminação positivos, variando de 3,0 a 16,7; consistência interna satisfatória com um alfa de Cronbach de ,75; e a estrutura fatorial com cinco fatores específicos: 1) Enfrentamento $e$ Autoafirmação com Risco - composto por onze itens que remetem a situações interpessoais que levam o respondente a reagir pela afirmação e defesa de direitos e autoestima, com risco potencial de reação indesejável ( $\alpha=.96$ como índice de confiabilidade desse fator); 2) Autoafirmação na Expressão de Afeto Positivo - composto por sete itens que apontam demandas interpessoais de expressão de afeto positivo e de afirmação de autoestima, possibilitando risco mínimo de situação indesejável - $(\alpha=, 86)$; 3) Conversação e Desenvoltura Social - formado também por sete itens que avaliam a capacidade de lidar com situações sociais neutras, com um risco mínimo de reação indesejável pelo outro - $(\alpha=, 81)$; 4) Autoexposição a Desconhecidos ou a Situações Novas - reúne quatro itens que envolvem pequeno risco de reação indesejável do interlocutor $-(\alpha=, 75) ; 5)$ Autocontrole da Agressividade compõe-se de 3 itens - composto por três itens que remetem a situações adversas com razoável controle da raiva e da agressividade - $(\alpha=, 74)$. Em estudo realizado por A. Del Prette, Del Prette e Barreto (1999) constatou-se que o IHS foi sensível para identificar modificações em estudantes universitários, com maiores escores das habilidades sociais no grupo treinado e ausência de efeito no grupo controle. Mostrou também a estabilidade temporal, uma vez que foi feito teste e re-teste no grupo controle. No estudo realizado por Bandeira, Z. Del Prette, Del Prette e Gerk-Carneiro (2000) em que estudantes de psicologia responderam ao IHS e à Escala de Assertividade de Rathus foi identificada correlação significativa entre as duas escalas. Na aplicação teste-reteste foi encontrada correlação significativa entre as duas aplicações, confirmando a estabilidade temporal do instrumento.

- A Escala Modos de Enfrentamento de Problemas (EMEP) (Vitaliano, Russo, Carr, Maiuro, \& Becker, 1985, adaptado para o português por Gimenes e Queiroz, 1997 e validado para a população brasileira por Seidl, Trocoli e Zannon, (2001) é um instrumento de autorrelato que conta com 45 itens distribuídos em quatro fatores que alcançam pensamentos e ações que o sujeito utiliza para lidar com as demandas externas e internas numa situação estressante: 1Enfrentamento Focalizado no Problema (18 itens, $\alpha=, 84$ como índice de confiabilidade desse fator); 2 - Enfrentamento Focalizado na Emoção (15 itens, $\alpha=, 81) ; 3$ - Busca de Suporte Social (5 itens, $\alpha=$,70); $4-$ Busca de Práticas Religiosas (7 itens, $\alpha=, 74$ ). As respostas são dadas em escala tipo Likert de 5 pontos (variando de 1 = "nunca faço isso" a 5 = "faço isso sempre"). Os escores são obtidos pela média aritmética e, quanto mais altos, maior a frequência de utilização da estratégia de enfrentamento.

- Escala de Habilidades para a Resolução de Problemas (EHRP) - (D’Zurilla \& Nezu, 1990 adaptada por Sá, 2005). Determina a capacidade de resolução de problemas de acordo com as habilidades comportamentais e cognitivas do sujeito. É composta por 40 itens distribuídos igualmente em quatro dimensões: 1) Formação de Problemas (10 itens, $\alpha=, 76$ como índice de confiabilidade desse fator), 2) Geração de Alternativas (10 itens, $\alpha=44)$, 3) Tomada de Decisões (10 itens, $\alpha=, 64)$ e 4) Verificação de Soluções (10 itens, $\alpha=, 57)$. O estudo Padovani, Schelini e Williams (2009) demonstrou que o EHRP foi efetivo para predizer o desempenho do grupo analisado, de adolescentes infratores e não infratores considerando o contexto brasileiro com propriedades psicométricas satisfatórias (Alpha de Cronbach de ,78 e teste-reteste de ,74).

\section{Procedimento}

A proposta da pesquisa foi aprovada pelo Comitê de Ética da Universidade com o consentimento voluntário dos participantes por meio da assinatura do Termo de Consentimento Livre e Esclarecido (TCLE). A aplicação dos instrumentos aconteceu em diferentes ambientes nas 
instituições de Ensino Superior, sendo que a maioria em salas de aula, coletivamente, dentro do horário combinado previamente com os professores. A ordem de aplicação dos instrumentos foi EHRP, IHS e EMEP em tempo médio de participação de 60 minutos.

\section{Análise de Dados}

A análise estatística descritiva de dados foi realizada com o software Statistical Package for the Social Sciences (SPSS) versão 20.0. Para verificar o impacto das variáveis explicativas Estratégias de Enfrentamento e Habilidades Sociais, seus respectivos fatores e escores totais, são preditoras da variável critério Resolução de Problemas foi utilizada a Regressão Linear Múltipla (RLM) cujos pressupostos foram testados pela normalidade (os desvios da pontuação de cada observação prevista na variável resposta em relação a pontuação verdadeira, que são normalmente distribuídas), independência (saber se as observações em um conjunto de dados estão correlacionadas umas com as outras) e multicolinearidade (que ocorre quando as variáveis explicativas estão altamente correlacionadas umas com as outras) entre os regressores e, em seguida, procedeu-se a análise de regressão linear múltipla (método Stepwise Forward).

\section{Resultados}

Os dados descritivos de cada um dos fatores das variáveis estudadas são apresentados na Tabela 1, com indicações de média e desvio-padrão disponíveis da amostra.

Tabela 1

Estatísticas descritivas para cada uma das variáveis

\begin{tabular}{clcc}
\hline Variáveis & \multicolumn{1}{c}{ Fatores } & Média & $D P$ \\
\hline & EAAR & 23,6 & 7,4 \\
Habilidades Sociais & AAESP & 18,7 & 4,1 \\
& CDS & 12,2 & 5,5 \\
& AEDSN & 7,8 & 2,9 \\
& ACA & 7,1 & 2,1 \\
\hline \multirow{2}{*}{ Estratégias de Enfrentamento de Problemas } & FP & 65,1 & 10,9 \\
& FE & 42,8 & 7,9 \\
& PR_PF & 22,4 & 5,3 \\
Habilidade para Resolução & BSS & 16,7 & 3,8 \\
\hline de Problemas & SDFP & 27,2 & 7,1 \\
& STD & 25,7 & 6,5 \\
& SGA & 25,4 & 6,8 \\
& SIVS & 24,3 & 6,3 \\
\hline
\end{tabular}

Nota: EAAR= Enfrentamento e autoafirmação com risco, AAESP= Autoafirmação na expressão de afeto positive, $\mathrm{CDS}=$ Conversação e desenvoltura social, AEDSN= Autoexposiçãoa desconhecidos, ACA= Autocontrole da agressividade, $\mathrm{FP}=$ Foco no problema, FE= Foco na emoção, PR_PF=Pensamento fantasioso, BSS=Suporte social, SDFP=Formação de problemas, STD=Tomada de decisão, SGA=Geração de alternativas e SIVS=Verificação de soluções. 
A Tabela 2 apresenta os fatores das variáveis preditoras para a relação de gerenciamento de situações sociais acadêmicas do aluno de Psicologia em seu cotidiano. O modelo significativo apresentado indica como os escores de estratégias de enfrentamento e habilidades sociais impactam nos escores de resolução de problemas. Os fatores Enfrentamento e autoafirmação com risco do IHS e Foco no Problema da EMEP, com $\beta=0,42$, explicam $25 \%$ da variância do fator Formação do Problema da EHRP (F= 6,84; $p=, 00)$. Em seguida, os fatores Foco no Problema, Foco na Emoção, Suporte Social e Pensamento Fantasioso da EMEP, juntamente com o fator Enfrentamento Autoafirmação com Risco do IHS, com $\beta=0,31$, explicam $21 \%$ da variância do fator Tomada de Decisão da EHRP $(\mathrm{F}=6,09 ; p=, 01)$. Posteriormente, os fatores Foco no Problema da EMEP, Enfrentamento Autoafirmação com Risco e Autoafirmação Afeto Positivo do IHS, com $\beta=0,39$, explicam $25 \%$ da variância do escore do fator Geração de Alternativas da EHRP (F= $7,38 ; p=, 01)$. Na sequência, dois fatores Autocontrole da Agressividade do IHS e Foco no Problema da EMEP, $\operatorname{com} \beta=0,35$, explicam $20 \%$ da variância do fator Verificação de Soluções da $\operatorname{EHRP}(\mathrm{F}=5,21 ; p=, 02)$.

Constata-se na Tabela 3 que o resultado da RLM mostrou que os escores gerais da Escala Modos de Enfrentamento de Problemas e do Inventário de Habilidades Sociais juntos explicam 16\% da variabilidade do escore geral da Escala de Habilidades de Resolução de Problemas. 
Tabela 2

Resultado de Análise de Regressão Linear Múltipla (RLM)

\begin{tabular}{|c|c|c|c|}
\hline & Beta & $t$ & $p$ \\
\hline IHS_EAARisco & 0,14 & 12,92 &, 00 \\
\hline EMEP_Foco Probl & $0,42 *$ & 2,62 & ,01 \\
\hline \multicolumn{4}{|c|}{ RLM para o fator "Tomada de Decisão" da EHRP. } \\
\hline & Beta & $t$ & $p$ \\
\hline EMEP_FocoProbl & $0,31 *$ & 8,59 & ,00 \\
\hline EMEP_FocoEmo & $-0,08$ & $-2,49$ & 01 \\
\hline IHS_DSocial & $-0,10$ & $-3,05$ &, 00 \\
\hline EMEP_SSocial & 0,09 & 2,74 &, 01 \\
\hline EMEP_PFantasi & $-0,08$ & $-2,47$ & 01 \\
\hline \multicolumn{4}{|c|}{ RLM para o fator "Geração de Alternativas" da EHRP } \\
\hline & Beta & $t$ & $p$ \\
\hline EMEP_FocoProbl & $0,39 *$ & 11,09 & 00 \\
\hline IHS_EAARisco & 0,07 & 2,09 &, 04 \\
\hline IHS_AAESPositv & 0,09 & 2,72 &, 01 \\
\hline \multicolumn{4}{|c|}{ RLM para o fator "Verificação de Soluções" da EHRP } \\
\hline & Beta & $t$ & $p$ \\
\hline EMEP_Foco Probl & $0,35^{*}$ & 10,34 & 00 \\
\hline IHS_ACAgressiv & $-0,09$ & $-3,14$ &, 00 \\
\hline
\end{tabular}

Nota: EMEP_FocoProbl = Escala Enfrentamento de Problema- Foco no Problema; EMEP_SSocial = Escala Enfrentamento de Problema - Suporte Social; EMEP_TOTAL = Escala Enfrentamento de Problema; IHS_AAESPositv = Inventário Habilidades Sociais - Autoafirmação Afeto Positivo; IHS_ACAgressiv = Inventário Habilidades Sociais - Autocontrole da Agressividade; IHS_DSocial= Inventário Habilidades Sociais - Desenvoltura Social; IHS_EAARisco = Inventário Habilidades Sociais - Enfrentamento Autoafirmação com Risco;IHS_TOTAL = Inventário Habilidades Sociais.

Tabela 3

Impacto dos escores totais de EMEP e IHS no escore total EHRP

\begin{tabular}{lcccccc}
\hline \multicolumn{1}{c}{ EHRP } & \multicolumn{2}{c}{ Coeficientes não-padronizados } & $\begin{array}{l}\text { Coeficientes } \\
\text { padronizados }\end{array}$ & $t$ & Sig. & $\mathrm{R}^{2}$ \\
\hline (Constant) & $\mathrm{B}$ & $\begin{array}{l}\text { Erro } \\
\text { padrão }\end{array}$ & Beta & & & \\
EMEP_TOTAL & 8,52 & 6,15 & - & 1,39 &, 16 & 0,16 \\
IHS_TOTAL & 0,39 & 0,04 & 0,32 & 9,83 &, 00 & \\
\hline
\end{tabular}




\section{Discussão}

Os coeficientes do modelo de regressão linear apreciados demonstraram (Tabela 2) que os fatores relacionados a situação interpessoal que leva o alunado a apresentar um risco potencial de reação indesejável (IHS_EAARisco) e a modificação da situação geradora de estresse (EMEP_Foco Probl) tiveram impacto positivo no fator "Formação do Problema" da EHRP, que aborda aspectos como aglutinação para o estabelecimento de metas realistas e resolutivas. Esse resultado aponta que provavelmente universitários que identificam suas demandas para superar as dificuldades, numa perspectiva cognitiva e/ou de enfrentamento, também se mostram mais focados em compreender e modificar sua situação problema, mesmo com risco de reação indesejável. Esse resultado encontra apoio na literatura (Sagone, \& De Caroli, 2014), visto que os universitários que demonstram utilizar a estratégia de enfrentamento Focada no Problema e percebem sua real situação, geralmente, elegem uma melhor forma para enfrentar/resolver problemas nas situações estressoras. Para Ranjbar et al. (2013) fazer uso de estratégia de enfrentamento no cotidiano pode favorecer a construção de um ambiente acadêmico mais harmonioso.

No que compreende os fatores relacionados à possibilidade de melhor manejo das situações estressoras (EMEP_FocoProbl) e a busca de cooperação e consolo de pessoas no seu meio social (EMEP_SSocial) observou-se impacto positivo no fator "Tomada de Decisão" da EHRP, que define a comparação de consequências das diferentes soluções para um problema. Esse dado evidencia que o uso da estratégia de suporte social, associada ao apoio encontrado nas pessoas e no ambiente pode reduzir o estresse, oferecendo condições propícias a racionalização dos problemas típicos do ambiente universitário e a avaliação da melhor opção de reposta. A percepção de amizade e cooperação, tanto no contexto acadêmico quanto fora dele, pode favorecer o discente na tomada de decisão (A. Del Prette \& Del Prette, 2017). Nota-se, desta forma, que as estratégias de foco no problema e suporte social podem ser consideradas adaptativas, ou seja, utilizadas para o afastamento e/ou enfrentamento do problema no processo de tomada de decisão (CórdobaSánchez \& Limonero-García, 2015). Constata-se também o impacto negativo dos fatores relacionados à tentativa de aliviar, diminuir ou regular o estado emocional do universitário diante de eventos estressantes (EMEP_FocoEmo), a uma postura de esquiva ou de negação diante da situação estressora (EMEP_Pfantasi) e a capacidade de lidar com situações sociais neutras (IHS_Dsocial) no fator “Tomada de Decisão” da EHRP. Estudantes que apresentam um repertório baseado na falta de controle emocional, na ausência de traquejo social ou que dependem de outros para resolução de alguma questão, ou mesmo aguardam que "forças ocultas" dissipem seus problemas, não se encontram em condições de tomar decisões adequadas (Ranjbar et al., 2013). Esse tipo de enfrentamento (com foco na emoção e pensamento fantasioso) pode representar uma reação paliativa para reduzir temporariamente a tensão causada pelo problema (Carlotto et al., 2015).

No que tange os fatores relacionados ao esforço ativo voltado para a reestruturação do problema (EMEP_FocoProbl), expressão de afeto positivo (IHS_AAESPositv) e a reação em defesa de direitos com risco potencial de reação indesejável (IHS_EAARisco), notou-se um impacto positivo no fator "Geração de Alternativas", que se refere a identificação do número variado de soluções. Tal dado sugere que o aluno que é assertivo na interação social é capaz de superar as dificuldades colocadas pela vida acadêmica. Esse resultado se aproxima dos achados de Pereira, Wagner e Oliveira (2014), que afirmam que estudantes de psicologia que já possuem um bom repertório para lidar com a expressão de sentimentos positivos, afirmação da autoestima e lidar com situações interpessoais que demandam afirmação e com a expressão de sentimentos positivos podem estar mais capacitados para resolver seus problemas. Tal fato corrobora o estudo de Hirsch et al. (2015) sobre a importância de se investigar como o graduando enfrentam situações adversas no contexto acadêmico. 
Em relação ao fator relacionado ao esforço voltado para a reavaliação do problema (EMEP_Foco Probl) verificou-se impacto positivo no fator "Verificação de Soluções", que compreende a avaliação os resultados das soluções escolhidas. Esse resultado mostra que o estudante que assume condutas de aproximação em relação ao estressor no sentido de solucionar o problema, consegue manejar melhor com a situação estressora. $\mathrm{O}$ fator que focaliza o problema refere-se ao empenho cognitivo e comportamental e funciona como um indicador de resposta positiva na resolução de problemas para enfrentar as situações adversas (Sagone \& Caroli, 2014). Ao focar o problema o universitário administra melhor a origem de suas questões e avalia seus efeitos, corroborando os dados da pesquisa de Padovani, Schelini e Williams (2009). Constatouse ainda impacto negativo do fator relacionado a situações adversas com razoável controle da raiva e da agressividade (IHS_ACAgressiv) no fator Verificação de Soluções. Tal resultado revela que o aluno que apresenta déficit no repertório no controle da Agressividade, dificilmente irá se comportar de forma socialmente competente para dissipar qualquer problema (Z. Del Prette, \& Del Prette, 2001). Esse é um dado preocupante, pois é necessário que os estudantes de psicologia tenham habilidades sociais para se comunicar com precisão, clareza e eficiência e, sobretudo, que possam controlar sua agressividade e encontrar soluções para seus desafios, bem como transformar conhecimento em uma prática efetiva (Pasqualotto et al., 2015) que é fundamental aos universitários em todas as configurações.

Cabe destacar (Tabela 2) que a estratégia de enfrentamento Focado no Problema do EMEP apresentou impacto em todos os fatores (Formação de Problema, Tomada de Decisão, Geração de Alternativas e Verificação de Soluções) da Escala de Habilidade de Resolução de Problemas Sociais. Todos esses fatores da EHRP podem afetar as percepções específicas de novas situações problemáticas, influenciar no tempo e no esforço que é dispendido, nas emoções que são geradas e na eficiência do desempenho dos sujeitos (D'Zurilla \& Nezu, 2007). A estratégia que focaliza o Problema refere-se ao empenho cognitivo e comportamental para enfrentar as situações de difícil manejo (Sagone, \& De Caroli, 2014) e é um indicador de resposta positiva de maior eficácia na resolução de problemas, com uma tendência adaptativa em processo cognitivo, visando o problema como possível de ser solucionado. A estratégia com foco no Problema pode envolver negociação para solução de uma situação de estresse interpessoal na resolução de um problema. Nota-se que o estudante de psicologia utiliza essa estratégia para definir um plano de ação, isto é, um conjunto de processos cognitivos para tentar reduzir o desconforto emocional, atenção seletiva e extração de valores positivos perante acontecimentos negativos para resolver uma situação adversa (Hirsch et al., 2015).

Ressalta-se que os alunos com habilidades para resolver problemas possuem estratégias de enfrentamento para lidar com situações difíceis/estressantes no contexto universitário que o prepara para a transição para o mercado de trabalho (Mourão, \& Abbad, 2016), o que pode ser crucial para a formação do aluno para um futuro bom desempenho social/laboral (Oliveira et al., 2014). O estudo apontou também que as estratégias de enfrentamento e as habilidades sociais juntas explicaram 16\% na resolução de problemas dos alunos ao contexto universitário (Tabela 3), ou seja, o aluno que tem um repertório mais elaborado de habilidades sociais e estratégias de enfrentamento mais realistas focadas objetivamente no problema tende a solucionar melhor seus problemas. Vale ressaltar que, embora o coeficiente de determinação ajustado possa parecer baixo (R2 ajustado $=0,16)$, é comum encontrar valores dessa ordem em casos práticos de análise de Regressão Linear Múltipla nas Ciências Sociais. Por exemplo, os estudos de Soares, Santos, Andrade e Souza (2017) que obtiveram R2 ajustado de 4,8\%. Esse resultado sinaliza que solucionar problemas está significativamente associado a um repertório elaborado de habilidades sociais (Ranjbar et al., 2013) para gerenciar situações sociais acadêmicas. Essa capacidade social de resolução de problemas envolve processos cognitivos e comportamentais necessários para lidar adequadamente com vários problemas estressantes experimentados no contexto acadêmico (D’Zurilla \& Nezu, 1990). Desta forma, o aluno que consegue identificar uma situação de conflito 
no âmbito acadêmico deve inevitavelmente se posicionar com firmeza para se recuperar de situações de crise, bem como superar os fatores estressores que vivencia consigo mesmo, características cognitivas, desde que tenha também apoio, suporte de outras pessoas. Isto é, o aluno relaciona as experiências positivas que os levam a sentimentos de autonomia, para que consiga lidar com mudanças para a solução de problemas. Assim, o estudante pode construir recursos pessoais que propiciam maior satisfação de como lidar com as adversidades (Juliano \& Yunes, 2014).

\section{Conclusão}

Este estudo procurou investigar a existência de relação preditiva entre os repertórios de habilidades sociais, estratégias de enfrentamento e resolução de problemas em estudantes de psicologia. Tendo em vista os estudos arrolados, constatou-se que os construtos enfrentamento de problemas e habilidades sociais juntos proporcionam consideravelmente melhores resultados na forma como os estudantes resolvem seus problemas.

O estudo constatou especialmente que os estudantes de psicologia dessa amostra apresentaram associação preditiva entre estratégia de enfrentamento e resolução de problema o que pode indicar que quanto maior a utilização de estratégias como papel essencial no desenvolvimento pessoal, social e acadêmico em situações problema, melhor a capacidade geral da tomada de decisões. Ainda que o presente estudo tenha proporcionado contribuição para o ajustamento da programação de ensino voltada para o desenvolvimento de habilidade de resolução de problemas envolvida na formação do futuro psicólogo, algumas limitações foram evidenciadas, como o número reduzido de estudantes de instituições públicas e de uma única região. Nesse sentido, faz-se necessária a realização de mais estudos com estagiários de psicologia, investigando dificuldades específicas de manejo de situações adversas. As habilidades sociais assertivas provavelmente podem explicar a solução de problemas dos alunos para lidar com as adversidades do contexto universitário, bem como identificar maneiras eficazes de resolver os problemas vivenciados pelos estudantes. No entanto, para ser capaz de intervenções realmente efetivas na melhoria dos déficits encontrados no ambiente acadêmico, é importante, inicialmente, o entendimento do desenvolvimento de habilidades sociais em estudantes universitários como requisito para o processo de aprendizagem e interação estagiário e paciente/cliente.

\section{Referências}

Bandeira, M., Costa, M. N., Del Prette, Z. A. P., Del Prette, A., \& Gerk-Carneiro, E. (2000). Qualidades psicométricas do Inventário de Habilidades Sociais (IHS): estudo sobre a estabilidade temporal e a validade concomitante. Estudos de Psicologia, 5(2), 401-419.

Carlotto, R. C., Teixeira, M. A. P., \& Dias, A. C. G. (2015). Adaptação acadêmica e coping em estudantes universitários. Psico-USF, 20(3), 421-432. doi: 10.1590/1413-82712015200305

Córdoba-Sánchez, V., \& Limonero-García, J. T. (2015). Coping and quality of life in patients with Systemic lupus erythematosus: a review. Pensando Psicología, 11(18), 129-139. doi: 10.16925/pe.v11i18.1223

Del Prette, A., \& Del Prette, Z. A. P. (2001). Inventário de Habilidades Sociais - IHS. São Paulo: Editora Casa do Psicólogo.

Del Prette, A., Del Prette, Z. A., \& Barreto, M. C. M. (1999). Habilidades sociales en la formación profesional del psicólogo: análisis de un programa de intervención. Psicología Conductual, 7(1), 27-47.

D'Zurilla, T. J., \& Nezu, A. M. (1990). Development and preliminary evaluation of the Social Problem-Solving Inventory. Psychological Assessment: A Journal of Consulting and Clinical Psychology, 2(2), 156-163. doi: 10.1037/1040-3590.2.2.156 
D'Zurilla, T. J. \& Nezu, A. M. (2007). Problem-solving therapy: A positive approach to clinical intervention (3a. ed.). New York: Springer Publishing Company.

Gbenga, J. B. \& David, B. T. (2015). Influential determinants of capacity building to cope with stress among university students. Journal of Sports and Physical Education, 2(3), 48-53.

Gimenes, M.G.G. (1997). A teoria do enfrentamento e suas implicações para sucessos e insucessos em Psicologia. In: M. G. G. Gimenes \& M.H. Fávero (Orgs.), A mulher e o câncer (pp. 111147). Campinas: Editorial Psy.

Hirsch, C. D., Barlem, E. L. D., Almeida, L. K. D., Tomaschewski-Barlem, J. G., Figueira, A. B., \& Lunardi, V. L. (2015). Estratégias de coping de acadêmicos de enfermagem diante do estresse universitário. Revista Brasileira de Enfermagem, 68(5), 783-790. doi: 10.1590/0034-7167.2015680503i

Juliano, M. C. C., \& Yunes, M. A. M. (2014). Reflections on the social support network as a mechanism for the protection and promotion of resilience. Ambiente \& Sociedade, 17(3), 135-154. doi: 10.1590/S1414-753X2014000300009

Leme, V. B. R., Del Prette, Z. A. P., Koller, S. H., \& Del Prette, A. (2016). Social Skills and Biocological Model of Human Development: Analyze and Perspectives. Psicologia \& Sociedade, 28(1), 181-193. doi: 10.1590/1807-03102015aop001

Lima, C. A., \& Soares, A. B. (2015). Treinamento em Habilidades Sociais para universitários no contexto acadêmico: ganhos e potencialidades em situações consideradas difíceis. Em Del Prette, Z. A. P., Soares, A. B., Pereira-Guizzo, C. S., Wagner, M. F., \& Leme, V. B. R. (Orgs). Habilidades Sociais: Diálogos e intercâmbios sobre pesquisa e prática (pp. 22-43). Novo Hamburgo, R. S. Sinopsys

Ministério da Educação. Instituto Nacional de Estudos e Pesquisas Educacionais Anísio Teixeira $\begin{array}{llllll}- & \text { ENADE } & - & \text { Curso } & \text { de }\end{array}$ download.inep.gov.br/educacao_superior/enade/relatorio_sintese/2015/psicologia.pdf

Monteiro, M. C., \& Soares, A. B. (2017). Conceptions of students on problem-solving and selfmonitoring. Psicologia Escolar e Educacional, 21(2), 333-341. doi: 10.1590/21753539201702121124

Mourão, L., \& Abbad, G. S. (2016). As lacunas de competências na formação em Psicologia e os riscos para a atuação profissional. Em A. B. Soares, L. Mourão \& M. M. P. E. Da Motta (Orgs.), O Estudante Universitário Brasileiro: Características cognitivas, habilidades relacionais e transição para o mercado de trabalho (pp. 318-334). Curitiba: Appris.

Oguz-Duran, N., \& Yuksel, A. (2010). The effects of coping humour and gender on college adjustment in Turkish freshmen. Education, 130(3), 470-479.

Oliveira, C. T., Carlotto, R, C., Vasconcelos, S. J. L. \& Dias, A. C. G. (2014). Adaptação acadêmica e coping em estudantes universitários brasileiros: Uma revisão de literatura. Revista Brasileira de Orientação Profissional, 15, 2, 177-186.

Padovani, R. D. C., Schelini, P. W., \& Williams, L. C. D. A. (2009). Inventário de Resolução de Problemas Sociais-Revisado: evidências de validade e precisão. Avaliação Psicológica, 8(2), 267-276.

Pasqualotto, R. A., Löhr, S. S., \& Stoltz, T. (2015). Skinner and Vygotsky's Understanding of Resilience in the School Environment. Creative Education, 6(17), 1841-1851. doi: 10.4236/ce.2015.617188

Pereira, A. S., Wagner, M. F., \& Oliveira, M. D. S. (2014). Déficits em habilidades sociais e ansiedade social: Avaliação de estudantes de Psicologia. Psicologia da Educação, 38, 113122.

Ramos, C. C., Costa, T. D., \& Feitosa, I. O. (2017). Mapeamento de incoerências entre competências estabelecidas na formação do psicólogo organizacional e as requeridas pelo mercado de trabalho. Revista Psicologia Organizações e Trabalho, 17(2), 114-120. doi: 10.17652/rpot/2017.2.12866 
Ranjbar, M., Bayani, A. A., \& Bayani, A. (2013). Social Problem Solving Ability Predicts Mental Health Among Undergraduate Students. International Journal of Preventive Medicine, 4(11), 1337-1342.

Reeve, K. L., Shumaker, C. J., Yearwood, E. L., Crowell, N. A., \& Riley, J. B. (2013). Perceived stress and social support in undergraduate nursing students' educational experiences. Nurse Education Today, 33(4), 419-424. doi: 10.1016/j.nedt.2012.11.009

Sá, A. P. (2005). A Importância do Psicodiagnóstico Diferencial dos Transtornos de Personalidade no Tratamento dos Transtornos de Ansiedade. Trabalho apresentado no V Congresso da Sociedade Brasileira de Terapias Cognitivas. Resumo recuperado de http://cbtc.fbtc.org.br/\#/ficha/2005

Sagone, E., \& De Caroli, M. E. (2014). A correlational study on dispositional resilience, psychological well-being, and coping strategies in university students. American Journal of Educational Research, 2(7), 463-471. doi: 10.12691/education-2-7-5

Santos, D. B. A. D. (2016). Qualidade de vida, estratégias de coping e bem-estar psicológico em estudantes universitários (Dissertação de Mestrado). Recuperado de http://hdl.handle.net/10437/7965

Seidl, E. M. F., Tróccoli, B. T., \& Zannon, C. M. L. C. (2001). Análise fatorial de uma medida de estratégias de enfrentamento. Psicologia: Teoria e Pesquisa, 17(3), 225-234. doi: 10.1590/S0102-37722001000300004

Soares, A. B., \& Prette, Z. A. P. D. (2015). Habilidades sociais e adaptação à universidade: Convergências e divergências dos construtos. Análise Psicológica, 33(2), 139-151. doi: 10.14417/ap.911

Soares, A. B., Gomes, G., Maia, F. D. A., Gomes, C. A. O., \& Monteiro, M. C. (2016). Relações interpessoais na universidade: O que pensam estudantes da graduação em Psicologia? Estudos Interdisciplinares em Psicologia, 7(1), 56-76. doi: 10.5433/2236-6407.2016v7n1p56

Soares, A. B., Santos, Z. A., Andrade, A. C., \& Souza, M. S. (2017). Expectativas académicas y habilidades sociales en la adaptación a la universidad. Ciencias Psicológicas, 11(1), 77-88. doi: 10.22235/cp.v11i2.1349

Tavares, W. M., Couto, G., \& Silva, R. L. F. C. (2012). Perfil de relações interpessoais e habilidades sociais de estudantes de Psicologia. Estudos Interdisciplinares em Psicologia, 3(1), 75-92.

Vitaliano, P. P., Russo, J., Carr, J. E., Maiuro, R. D., \& Becker, J. (1985). The ways of coping checklist: Revision and psychometric properties. Multivariate Behavioral Research, 20(1), 3 26. doi: 10.1207/s15327906mbr2001_1

Participação dos autores: a) Planejamento e concepção do trabalho; b) Coleta de dados; c) Análise e interpretação de dados; d) Redação do manuscrito; e) Revisão crítica do manuscrito.

Z.A.S. contribuiu em a, b, c, d; A.B.S. em e.

Editora científica responsável: Dra. Cecilia Cracco 